\title{
Convergence of Galerkin Approximations for the Korteweg-de Vries Equation*
}

\author{
By Garth A. Baker, Vassilios A. Dougalis and Ohannes A. Karakashian
}

\begin{abstract}
Standard Galerkin approximations, using smooth splines on a uniform mesh, to 1-periodic solutions of the Korteweg-de Vries equation are analyzed. Optimal rate of convergence estimates are obtained for both semidiscrete and second order in time fully discrete schemes. At each time level, the resulting system of nonlinear equations can be solved by Newton's method. It is shown that if a proper extrapolation is used as a starting value, then only one step of the Newton iteration is required.
\end{abstract}

1. Introduction. This work is aimed at deriving rate of convergence estimates for standard Galerkin approximations, using smooth splines on a uniform mesh, to 1-periodic solutions of the Korteweg-de Vries equation.

For $0<T<\infty$, a function $u:[0,1] \times[0, T] \rightarrow R$ is sought satisfying

$$
\left\{\begin{array}{l}
\frac{\partial u}{\partial t}+u \frac{\partial u}{\partial x}+\frac{\partial^{3} u}{\partial x^{3}}=0, \quad \text { on }(0,1) \times(0, T], \\
\frac{\partial^{j} u}{\partial x^{j}}(0, t)=\frac{\partial^{j} u}{\partial x^{j}}(1, t), \quad j=0,1,2 ; t \in[0, T], \\
u(x, 0)=u^{0}(x), \quad x \in[0,1],
\end{array}\right.
$$

where $u^{0}(x)$ is a given 1-periodic function.

It will be assumed that (1.1) has a unique solution, sufficiently smooth to guarantee the convergence results below. Results on existence, uniqueness and regularity of solutions of this problem have been obtained, e.g., in [3], [4], [7], [10].

A nonstandard dissipative Galerkin method yielding the optimal rate of convergence for the semidiscrete approximation for (1.1) using arbitrary $C^{2}(0,1)$ periodic piecewise polynomial functions has been analyzed by Wahlbin [14]. A nonstandard Galerkin method, yielding optimal rate of convergence estimates for both semidiscrete and second order accurate in time fully discrete schemes has been analyzed by Winther [15].

For numerical work concerning the Korteweg-de Vries equation, cf. Alexander and Morris [2] for dissipative and nondissipative Galerkin approximations to (1.1). For finite difference schemes cf. e.g. [6], [13], [17]. For spectral methods cf. e.g. [1], [5], the appendix of [7], [8], [9]. See also the references of [16].

It is stated in [14] that the semidiscrete approximation for the standard Galerkin method using smooth periodic splines of order $r \geqslant 4$ gives the optimal convergence

Received October 31, 1980; revised August 19, 1981, March 10, 1982 and June 21, 1982.

1980 Mathematics Subject Classification. Primary 65N30; Secondary 35Q20, 65M15.

* Research supported by ONR (jrant No. 33-720-7303-2 and USARO Grant No. DAA(;29-80-K-0056. 
rate $O\left(h^{r}\right)$ in $L^{2}(0,1)$. For the convenience of the reader we supply a proof of this here. We use some key estimates of Thomée and Wendroff [12] for some associated linearized problems. We then analyze a fully discrete Crank-Nicolson type approximation to $u$. Among the results obtained are the existence, uniqueness, stability as well as the optimal convergence rate of the fully discrete approxirnation. At each time level, the resulting system of nonlinear equations can be solved by Newton's method. It is shown that if a proper extrapolation is used as a starting value, then only one step of the Newton iteration is required to preserve the stability and optimal rate of convergence of the scheme.

Some of the results above hold provided conditions relating the sizes of $k$ and $h$, the time and space discretization parameters, respectively, are satisfied. Specifically, for the proof of uniqueness of the solution of the fully discrete Crank-Nicolson scheme, a condition of the form $k h^{-1 / 2}<c$, with sufficiently small $c$ independent of $k$ and $h$, must hold. Also, for the convergence of Newton's method, a condition of the form $k h^{-3 / 4}<c$, $c$ sufficiently small, is required. We point out that this is a very weak limitation on the time step $k$ and is certainly satisfied in practice. Note that most finite-difference schemes, cf. e.g. [13], [17], require a severe restriction on $k$ of the form $k h^{-3}<c$. However Winther's [15] scheme is unconditionally stable.

An outline of the paper is as follows: Section 2 is devoted to establishing notation and the statements, in the form of theorems, of the results obtained. In Section 3, the proofs of those results are given.

2. Rate of Convergence Estimates. We next establish the notation to be used throughout the paper and state the main results obtained; the proofs will be given in Section 3.

For real $s$ and $1 \leqslant p \leqslant \infty, W_{p}^{s}(0,1)$ will denote the Sobolev space of real valued functions on $(0,1)$, the norm of which we denote by $\|\cdot\|_{W_{p}^{s}(0,1)}$. For convenience, for $p=2$ we shall write $\|\cdot\|_{s}=\|\cdot\|_{W_{2}^{5}(0,1)}$. The inner product and norm on $W_{2}^{0}(0,1) \equiv$ $L^{2}(0,1)$, we denote by $(\cdot, \cdot)$ and $\|\cdot\|$, respectively. For $X$ a normed linear space with norm $\|\cdot\|_{X}$, and $u:[0, T] \rightarrow X$ measurable, we define

$$
\|u\|_{L^{p}(0, T ; X)}=\left\{\int_{0}^{T}\|u(t)\|_{X}^{p} d t\right\}^{1 / p}, \quad 1 \leqslant p<\infty,
$$

and

$$
\|u\|_{L^{\infty}(0, T ; X)}=\underset{0 \leqslant t \leqslant t}{\operatorname{ess} \sup }\|u(t)\|_{X} .
$$

For integer $r \geqslant 4, S_{h}^{r}$ will denote the space of 1-periodic smooth splines of degree $r-1$ on a uniform mesh of width $h=N^{-1}$ on $[0,1]$. $S_{h}^{r}$ possesses the well-known approximation properties:

If $v$ is 1-periodic and $v \in W_{2}^{s}(0,1) \cap W_{\infty}^{m}(0,1)$, then there exists a $\chi \in S_{h}^{r}$ such that

$$
\sum_{j=0}^{s-1} h^{j}\|v-\chi\|_{j} \leqslant c h^{s}\|v\|_{s}, \quad 1 \leqslant s \leqslant r
$$

and

$$
\sum_{j=0}^{m-1} h^{j}\|v-\chi\|_{W_{\infty}^{j}(0,1)} \leqslant c h^{m}\|v\| w_{\infty}^{m}(0,1), \quad 1 \leqslant m \leqslant r .
$$


Also, for all $\chi \in S_{h}^{r}$, the following “inverse assumptions" hold:

$$
\begin{aligned}
& \|\chi\|_{\beta} \leqslant c h^{-(\beta-\alpha)}\|\chi\|_{\alpha}, \quad 0 \leqslant \alpha \leqslant \beta \leqslant r-1, \\
& \|\chi\|_{w_{\infty}^{s}(0,1)} \leqslant c h^{-(s+1 / 2)}\|\chi\|, \quad 0 \leqslant s \leqslant r-1 .
\end{aligned}
$$

$c$ above is independent of $h$ and throughout the paper will denote a generic constant, not necessarily the same in any two places.

The following theorem defines the semidiscrete Galerkin approximation to $u(x, t)$, the solution of (1.1), and gives an error estimate which has optimal rate of convergence. In what follows we let $P u^{0}$ denote any conveniently chosen element of $S_{h}^{r}$ (e.g. $L^{2}$-projection, interpolant, etc.) which satisfies, for sufficiently smooth $u^{0}$,

$$
\left\|P u^{0}-u^{0}\right\| \leqslant c h^{r} .
$$

THEOREM 2.1. There exists a unique mapping $v_{h}:[0, T] \rightarrow S_{h}^{r}$ satisfying

$$
\left\{\begin{array}{l}
\left(v_{h t}+v_{h} v_{h x}+v_{h x x x}, \chi\right)=0, \quad \forall \chi \in S_{h}^{r}, t \in(0, T], \\
v_{h}(0)=P u^{0}
\end{array}\right.
$$

Moreover, if $u$ is sufficiently smooth, then for some constant $c$ depending only on $u$ and $T$,

$$
\left\|u-v_{h}\right\|_{L^{\infty}\left(0, T ; L^{2}(0,1)\right)} \leqslant c h^{r} .
$$

For $J$ a positive integer, let $k$ be such that $k J=T$. For $v:[0, T] \rightarrow L^{2}(0,1)$ continuous, we define

$$
v^{n}=v(x, n k), \quad \partial v^{n}=k^{-1}\left(v^{n+1}-v^{n}\right)
$$

and $v^{n+1 / 2}=\frac{1}{2}\left(v^{n+1}+v^{n}\right)$, for $n=0,1, \ldots, J-1$. The Crank-Nicolson fully discrete approximation is defined in the following way: seek a sequence $\left\{U^{n}\right\}_{n=0}^{J} \subset S_{h}^{r}$ satisfying

$$
\left\{\begin{array}{l}
\left(\partial U^{n}+U^{n+1 / 2} U_{x}^{n+1 / 2}+U_{x x x}^{n+1 / 2}, \chi\right)=0, \quad \forall \chi \in S_{h}^{r}, n=0,1, \ldots, J-1 \\
U^{0}=P u^{0}
\end{array}\right.
$$

We have the following

THEOREM 2.2. There exists a sequence $\left\{U^{n}\right\}_{n=0}^{J}$ satisfying (2.6). Also, for $k, h$ sufficiently small and for some constant $c=c(u, T)$

$$
\max _{0 \leqslant n \leqslant J}\left\|u^{n}-U^{n}\right\| \leqslant c\left\{h^{r}+k^{2}\right\} .
$$

In addition, there exists a positive constant $\gamma_{0}=\gamma_{0}(u, T)$ such that if $k h^{-1 / 2}<\gamma_{0}$, then the sequence $\left\{U^{n}\right\}_{n=0}^{J}$ is unique.

We note that, by periodicity, $\left\{U^{n}\right\}_{n=0}^{J}$ can be equivalently defined by

$$
\left\{\begin{aligned}
\left(\partial U^{n}, \chi\right)-\frac{1}{2}\left(\left[U^{n+1 / 2}\right]^{2}, \chi_{x}\right)+\left(U_{x x x}^{n+1 / 2}, \chi\right)=0 \\
\forall \chi \in S_{h}^{r}, n=0, \ldots, J-1, \\
U^{0}=P u^{0}
\end{aligned}\right.
$$

Whenever convenient, either form shall be used.

We propose to solve the nonlinear equations (2.6') using Newton's method. In practical situations this process is terminated after a finite number of iterations. 
With this in mind, we define the following scheme: Let $\left\{j_{0}, j_{1}, \ldots, j_{J}\right\}$ be a collection of integers with $j_{n} \geqslant 1,0 \leqslant n \leqslant J$ (we can actually take $j_{n}=1$ ). Let $\tilde{U}_{j_{n}}^{n}$ be an approximation to $U^{n}$, and let $\tilde{U}^{n+1}$ be the exact solution of the nonlinear system (2.6') with $U^{n}$ replaced by $\tilde{U}_{j_{n}}^{n}$, i.e., let $\tilde{U}^{n+1}$ be given for $0 \leqslant n \leqslant J-1$ by

$$
\begin{aligned}
\left(\tilde{U}^{n+1}-\tilde{U}_{j_{n}}^{n}, \chi\right) & -\frac{k}{8}\left(\left[\tilde{U}^{n+1}+\tilde{U}_{j_{n}}^{n}\right]^{2}, \chi_{x}\right) \\
+ & \frac{k}{2}\left(\left[\tilde{U}^{n+1}+\tilde{U}_{j_{n}}^{n}\right]_{x x x}, \chi\right)=0, \quad \forall \chi \in S_{h}^{r},
\end{aligned}
$$

where we set

$$
\tilde{U}_{j_{0}}^{0}=U^{0}=P u^{0}
$$

Since we cannot solve (2.7) exactly, we shall approximate its solution using Newton's method. For this, let $\tilde{U}_{0}^{n+1}$ be an initial approximation to $\tilde{U}^{n+1}$ obtained in the following way: For $n=0, \tilde{U}_{0}^{1}$ is the solution of the linear system

$$
\left(\tilde{U}_{0}^{1}-U^{0}, \chi\right)+k\left(U^{0}\left[\tilde{U}_{0}^{1}\right]_{x}, \chi\right)+k\left(\left[\tilde{U}_{0}^{1}\right]_{x x x}, \chi\right)=0, \quad \forall \chi \in S_{h}^{r},
$$

and for $n \geqslant 1$

$$
\tilde{U}_{0}^{n+1}=2 \tilde{U}_{j_{n}}^{n}-\tilde{U}_{j_{n-1}}^{n-1} .
$$

Then Newton's method for obtaining $\tilde{U}_{j_{n+1}^{n+1}}^{n}$, an approximation to $\tilde{U}^{n+1}$, is:

$$
\begin{aligned}
&\left(\tilde{U}_{j+1}^{n+1}+\frac{k}{2}\left[\tilde{U}_{j+1}^{n+1}\right]_{x x x}, \chi\right)-\frac{k}{4}\left(\left[\tilde{U}_{j}^{n+1}+\tilde{U}_{j_{n}}^{n}\right] \tilde{U}_{j+1}^{n+1}, \chi_{x}\right) \\
&=-\frac{k}{8}\left(\left[\tilde{U}_{j}^{n+1}\right]^{2}-\left[\tilde{U}_{j_{n}}^{n}\right]^{2}, \chi_{x}\right)+\left(\tilde{U}_{j_{n}}^{n}-\frac{k}{2}\left[\tilde{U}_{j_{n}}^{n}\right]_{x x x}, \chi\right) \\
& \forall \chi \in S_{h}^{r}, 0 \leqslant j \leqslant j_{n+1}-1 .
\end{aligned}
$$

We have

THEOREM 2.3. Suppose $j_{n} \geqslant 1, \forall n$ and $h, k$ are sufficiently small. Then, there exists a constant $\gamma_{1}=\gamma_{1}(u, T)$ such that if $k h^{-3 / 4}<\gamma_{1}$, there exists a unique sequence $\left\{\tilde{U}_{j_{n}}^{n}\right\}_{n=0}^{J}$ given by (2.8), (2.9), (2.9') and (2.10). Moreover, there exists a constant $c$ independent of $h, k$ and the integers $j_{n}$ such that

$$
\max _{0 \leqslant n \leqslant J}\left\|U^{n}-\tilde{U}_{j_{n}}^{n}\right\| \leqslant c\left\{k^{2}+h^{r}\right\} .
$$

Thus Theorems 2.2 and 2.3 show that $\max _{0 \leqslant n \leqslant J}\left\|u^{n}-\tilde{U}_{j_{n}}^{n}\right\|=O\left(k^{2}+h^{r}\right)$.

3. Convergence Proofs. In [12], V. Thomée and B. Wendroff consider the following initial value problem $(D=\partial / \partial x)$ :

$$
\left\{\begin{array}{l}
\frac{\partial u}{\partial t}=L(x, t, D) u \equiv \sum_{0 \leqslant \alpha \leqslant m} P_{\alpha}(x, t) D^{\alpha} u, \quad x \in R, t>0 \\
u(x, 0)=u^{0}(x) .
\end{array}\right.
$$

$m$ is a positive integer such that $r \geqslant(m+2) / 2$; the coefficients and the initial value are real-valued sufficiently smooth 1 -periodic functions in $x$. Moreover, the operator $L=L(x, t, D)$ is assumed to be semibounded, so that

$$
(L u, u) \leqslant c_{0}\|u\|^{2}, \quad t>0 .
$$


Writing the differential operator $L$ in the form

$$
L(x, t, D) u=\sum_{\substack{\alpha<r \\ \beta<r}} D^{\alpha}\left(P_{\alpha \beta} D^{\beta} u\right)
$$

they introduce the bilinear form,

$$
B(v, w)=\sum_{\alpha, \beta}(-1)^{\alpha}\left(P_{\alpha \beta} D^{\beta} v, D^{\alpha} w\right)
$$

and consider the Galerkin problem of finding $v(t) \in S_{h}^{r}$ such that

$$
\left\{\begin{array}{l}
\left(\frac{\partial v}{\partial t}, \chi\right)=B(v, \chi), \quad \forall \chi \in S_{h}^{r}, t>0, \\
v(0)=u_{h}^{0}
\end{array}\right.
$$

where $u_{h}^{0}$ is the quasi-interpolant of $u^{0}$ (cf. [12, p. 1062]). They obtain the following estimate for $u$ sufficiently smooth:

$$
\|v(x, t)-u(x, t)\| \leqslant c h^{r}, \quad 0 \leqslant t \leqslant T,
$$

where $c$ depends only on $u$ and $T$.

We consider the operator

$$
L=-u \frac{\partial}{\partial x}-\frac{\partial^{3}}{\partial x^{3}}
$$

where $u$ is the solution of (1.1). Now for $v \in W_{2}^{r-1}(0,1), 1$-periodic,

$$
(L v, v)=\frac{1}{2} \int_{0}^{1} u_{x} v^{2} d x \leqslant \frac{1}{2}\left\|u_{x}\right\|_{L^{\infty}(0,1)}\|v\|^{2}, \quad t>0 .
$$

Thus we have

LEMma 3.1. Let $u$ be the solution of (1.1), assumed to be sufficiently smooth; then there exists a unique function $w:[0, T] \rightarrow S_{h}^{r}$ satisfying

$$
\left\{\begin{array}{l}
\left(w_{t}+u w_{x}+w_{x x x}, \chi\right)=0, \quad \forall \chi \in S_{h}^{r}, t \in(0, T], \\
w(x, 0)=u_{h}^{0} .
\end{array}\right.
$$

Moreover, there exists a constant $c$, independent of $h$, such that

$$
\|u-w\|_{L^{\infty}\left(0, T: L^{2}(0,1)\right)} \leqslant c h^{r} .
$$

Proof of Theorem 2.1. We first consider the existence of a solution $v_{h}$ to (2.5). Let $\left\{\phi_{i}\right\}_{i=1}^{N}$ be a basis of $S_{h}^{r}$. We have

$$
v_{h}(x, t)=\sum_{j=1}^{N} y_{j}(t) \phi_{j}(x) .
$$

Using this, (2.5) is reduced to an initial value ODE problem

$$
Y_{t}=f(Y), \quad Y=\left\{y_{1}, \ldots, y_{N}\right\}^{T} .
$$

Using (2.3a) and (2.3b), it can be shown easily that $f$ is continuous. Moreover, by letting $\chi=v_{h}$ in (2.5), by periodicity we obtain

$$
\frac{d}{d t}\left\|v_{h}(t)\right\|^{2}=0
$$


hence

$$
\left\|v_{h}(t)\right\|=\left\|P u^{0}\right\|, \quad t \geqslant 0
$$

Now from (2.3b)

$$
\left\|v_{h}(t)\right\|_{L^{\infty}(0,1)} \leqslant c h^{-1 / 2}\left\|v_{h}(t)\right\|=c h^{-1 / 2}\left\|P u^{0}\right\|
$$

hence a unique solution $v_{h}(t)$ exists for $t>0$.

Now let $w$ be defined by (3.8). Let $e=v_{h}-w$ and $\theta=u-w$. From (2.5) and (3.8) we have

$$
\begin{aligned}
\left(e_{t}+e_{x x x}, \chi\right) & =\left(u w_{x}-v_{h} v_{h x}, \chi\right) \\
& =\left(-w e_{x}+\theta w_{x}-e e_{x}-e w_{x}, \chi\right), \quad \forall \chi \in S_{h}^{r}, t>0
\end{aligned}
$$

Letting $x=e$, by periodicity we have

$$
\begin{aligned}
\frac{1}{2} \frac{d}{d t}\|e(t)\|^{2} & =-\frac{1}{2}\left(w_{x}(t),[e(t)]^{2}\right)+\left(w_{x}(t) \theta(t), e(t)\right) \\
& \leqslant c\left\|w_{x}(t)\right\|_{L^{\infty}(0,1)}\left\{\|\theta(t)\|^{2}+\|e(t)\|^{2}\right\},
\end{aligned}
$$

where we have used the Schwarz and arithmetic-geometric mean inequalities. Now from (2.3b) we have $\forall \chi \in S_{h}^{r}$,

$$
\begin{aligned}
\left\|w_{x}(t)\right\|_{L^{\infty}(0,1)} \leqslant & \left\|u_{x}(t)\right\|_{L^{\infty}(0,1)}+\left\|u_{x}(t)-\chi_{x}\right\|_{L^{\infty}(0,1)}+\left\|\chi_{x}-w_{x}(t)\right\|_{L^{\infty}(0,1)} \\
\leqslant & \left\|u_{x}(t)\right\|_{L^{\infty}(0,1)}+\left\|u_{x}(t)-\chi_{x}\right\|_{L^{\infty}(0,1)}+c h^{-3 / 2}\|\chi-w(t)\| \\
\leqslant & \left\|u_{x}(t)\right\|_{L^{\infty}(0,1)}+\left\|u_{x}(t)-\chi_{x}\right\|_{L^{\infty}(0,1)} \\
& +c h^{-3 / 2}\{\|u(t)-\chi\|+\|u(t)-w(t)\|\}
\end{aligned}
$$

Now, from (2.1), (2.2) and using (3.9), we get

$$
\begin{aligned}
\left\|w_{x}(t)\right\|_{L^{\infty}(0,1)} \leqslant & \left\|u_{x}(t)\right\|_{L^{\infty}(0,1)}+c h^{r-1}\|u\|_{w_{\infty}^{r}(0,1)} \\
& +c h^{r-3 / 2}\|u\| w_{2}^{r}(0,1)+c h^{r-3 / 2}, \quad t>0 .
\end{aligned}
$$

Hence, since $r \geqslant 4$, we have

$$
\left\|w_{x}\right\|_{L^{\infty}\left(0, T ; L^{\infty}(0,1)\right)} \leqslant c=c(u, T) .
$$

Returning to (3.12), we obtain

$$
\frac{d}{d t}\|e(t)\|^{2}-c\|e(t)\|^{2} \leqslant c\|\theta(t)\|^{2} .
$$

The last inequality yields via Gronwall's Lemma that

$$
\|e\|_{L^{\infty}\left(0, T ; L^{2}(0,1)\right)} \leqslant c\left(\|\theta\|_{L^{2}\left(0, T ; L^{2}(0,1)\right)}+\|e(0)\|\right)
$$

where $c$ depends only on $u$ and $T$. The proof of the theorem now follows by the triangle inequality, (2.4), (3.9), and [12, Lemma 2.4].

We now turn to the proofs of the error estimates for the fully discrete schemes. To prove Theorems 2.2 and 2.3 we shall compare, for each $n$, e.g., $U^{n}$ with an appropriate function $u_{h}(n k)$ in $S_{h}^{r}$. It turns out that the solution $v_{h}$ of the semidiscretization (2.5) may not be suitable as such a comparison function since various necessary estimates of norms of some of its higher time derivatives seem hard to 
obtain. We shall use instead $u_{h}$, a suitable quasi-interpolant of $u$, defined for $(x, t) \in[0,1] \times[0, T]$ by

$$
u_{h}(x, t)=\sum_{j=1}^{N} u(j h, t) \tilde{\Phi}_{j}(x),
$$

where $\left\{\tilde{\Phi}_{j}\right\}_{j=1}^{N}$ is a suitably chosen basis of $S_{h}^{r}$ constructed as follows (cf. e.g. [12]).

Let $\phi=\chi^{* r}$ (the $r$-fold convolution), where $\chi$ is the characteristic function of $\left[-\frac{1}{2}, \frac{1}{2}\right]$ and let, for some nonnegative integer $s$ and constants $d_{j}, \tilde{\phi}(x)=$ $\sum_{j=-s}^{s} d_{j} \phi(x-j)$. Then define for $1 \leqslant j \leqslant N \tilde{\Phi}_{j}(x)$ to be the restriction to $[0,1]$ of the function $\Sigma_{l \in Z} \tilde{\phi}_{j+l N}(x)$. It is then well known, cf. e.g. [12, Lemma 2.4], that if the $d_{j}$ are suitably chosen, it follows that

$$
\left\|u_{h}(t)-u(t)\right\| \leqslant c h^{r}\left\|\frac{\partial^{r} u}{\partial x^{r}}(t)\right\|, \quad 0 \leqslant t \leqslant T,
$$

holds. We collect all additional results concerning $u_{h}$ that will be needed in sequel in the following

Lemma 3.2. Suppose $u$, the solution of (1.1), is sufficiently smooth. Then, for $h$ sufficiently small, there exists a constant $c$, independent of $h$, such that

$$
\left(u_{h t}+u u_{h x}+u_{h x x x}, \chi\right)=(\psi(t), \chi), \quad \forall \chi \in S_{h}^{r}, 0 \leqslant t \leqslant T,
$$

where

$$
\|\psi\|_{L^{\infty}\left(0, T ; L^{2}(0,1)\right)} \leqslant c h^{r} .
$$

Moreover we have the following bounds (with $D_{t}^{i}=\partial^{i} / \partial t^{i}$ ):

$$
\begin{gathered}
\left\|D_{t}^{i} u_{h}\right\|_{L^{\infty}\left(0, T ; L^{2}(0,1)\right)} \leqslant c, \quad i=0,1,2,3, \\
\left\|D_{t}^{i} u_{h}\right\|_{L^{\infty}\left(0, T ; W_{\infty}^{1}(0,1)\right)} \leqslant c, \quad i=0,1 .
\end{gathered}
$$

Proof. If the "truncation error" $\psi(t)$ is defined by (3.19), then (3.20) follows from Lemmas 2.2 and 4.2 of [12]. Since the quasi-interpolation operator commutes with time differentiation, (3.21) and (3.22) follow from (3.18), (2.1), (2.2) and (2.3a,b).

It can be seen that the exponent $r$ in (3.20) may be raised to the (superconvergent) value $\nu=2 r-2$ with a special choice of the basis $\left\{\tilde{\Phi}_{j}\right\}_{j=1}^{N}$ as is done in [12]. Here we only need that $\|\psi\|$ be of $O\left(h^{r}\right)$. It is also not hard to see that an alternative proof of Theorem 2.1 could have been given in which $v_{h}$ would have been compared with $u_{h}$ instead of $w$, the solution of (3.8).

Proof of Theorem 2.2. We shall first establish the rate of convergence estimates; so assume a sequence $\left\{U^{n}\right\}_{n=0}^{J} \subset S_{h}^{r}$ satisfying (2.6) exists. Letting $\chi=U^{n+1 / 2}$ in (2.6), we have by periodicity

$$
\frac{1}{2 k}\left\{\left\|U^{n+1}\right\|^{2}-\left\|U^{n}\right\|^{2}\right\}=-\left(U^{n+1 / 2} U_{x}^{n+1 / 2}+U_{x x x}^{n+1 / 2}, U^{n+1 / 2}\right)=0
$$

hence

$$
\left\|U^{n}\right\|=\left\|U^{0}\right\|, \quad n=0, \ldots, J
$$


Let $u_{h}$ be defined by (3.17). From (3.19), putting $\eta(t)=u_{h}(t)-u(t)$, we have

$$
\begin{aligned}
& \left(u_{h t}^{n+1 / 2}+\frac{1}{2}\left[u_{h}^{n+1} u_{h x}^{n+1}+u_{h}^{n} u_{h x}^{n}\right]+u_{h x x x}^{n+1 / 2}, \chi\right) \\
& \quad=\left(\psi^{n+1 / 2}+\frac{1}{2}\left[u_{h x}^{n} \eta^{n}+u_{h x}^{n+1} \eta^{n+1}\right], \chi\right), \quad \forall \chi \in S_{h}^{r}, 0 \leqslant n \leqslant J-1 .
\end{aligned}
$$

With $\zeta^{n}=U^{n}-u_{h}^{n}, n=0, \ldots, J$, a simple calculation using (3.25) and (2.6) gives, for $\chi \in S_{h}^{r}$,

$$
\begin{aligned}
& \left(\partial \zeta^{n}+\zeta_{x x x}^{n+1 / 2}, \chi\right)=\left(\partial U^{n}+U_{x x x}^{n+1 / 2}, \chi\right)-\left(\partial u_{h}^{n}+u_{h x x x}^{n+1 / 2}, \chi\right) \\
& =\left(u_{h t}^{n+1 / 2}-\partial u_{h}^{n}, \chi\right)-\left(U^{n+1 / 2} U_{x}^{n+1 / 2}, \chi\right) \\
& +\frac{1}{2}\left(u_{h}^{n+1} u_{h x}^{n+1}+u_{h}^{n} u_{h x}^{n}, \chi\right)-\left(\varepsilon^{n}, \chi\right) \\
& =\left(\rho^{n}+\delta^{n}-\varepsilon^{n}, \chi\right) \\
& -\left(\zeta^{n+1 / 2} \zeta_{x}^{n+1 / 2}+u_{h x}^{n+1 / 2} \zeta^{n+1 / 2}+u_{h}^{n+1 / 2} \zeta_{x}^{n+1 / 2}, \chi\right),
\end{aligned}
$$

where we have set

$$
\rho^{n}=u_{h t}^{n+1 / 2}-\partial u_{h}^{n}, \quad \varepsilon^{n}=\psi^{n+1 / 2}+\frac{1}{2}\left(u_{h x}^{n} \eta^{n}+u_{h x}^{n+1} \eta^{n+1}\right)
$$

and

$$
\delta^{n}=\frac{1}{4}\left[u_{h}^{n+1}-u_{h}^{n}\right]\left[u_{h x}^{n+1}-u_{h x}^{n}\right]
$$

Letting $\chi=\zeta^{n+1 / 2}$ in (3.26), we have by periodicity

$$
\begin{aligned}
& \left\|\zeta^{n+1}\right\|^{2}-\left\|\zeta^{n}\right\|^{2}=2 k\left(\rho^{n}+\delta^{n}-\varepsilon^{n}, \zeta^{n+1 / 2}\right)-k\left(u_{h x}^{n+1 / 2},\left[\zeta^{n+1 / 2}\right]^{2}\right) \\
& \leqslant k\left\{\left\|\zeta^{n+1}\right\|^{2}+\left\|\zeta^{n}\right\|^{2}+\left\|\rho^{n}\right\|^{2}+\left\|\delta^{n}\right\|^{2}+\left\|\varepsilon^{n}\right\|^{2}\right\} \\
& +\frac{k}{2}\left\|u_{h x}^{n+1 / 2}\right\|_{L^{x}(0.1)}\left\{\left\|\zeta^{n+1}\right\|^{2}+\left\|\zeta^{n}\right\|^{2}\right\} .
\end{aligned}
$$

Now, using (3.20), (3.22) and (3.18) we conclude that

$$
\max _{0 \leqslant n \leqslant J-1}\left\|\varepsilon^{n}\right\| \leqslant c h^{r} \text {. }
$$

Using (3.38) and (3.22) in (3.27), we get for some constants $c_{1}, c_{2}$ independent of $h$ and $k$

$$
\begin{aligned}
& \left(1-c_{1} k\right)\left\|\zeta^{n+1}\right\|^{2}-\left(1+c_{1} k\right)\left\|\zeta^{n}\right\|^{2} \\
& \leqslant c_{2} k\left\{\left\|\rho^{n}\right\|^{2}+\left\|\delta^{n}\right\|^{2}+h^{2 r}\right\}, \quad 0 \leqslant n \leqslant J-1,
\end{aligned}
$$

from which it follows that

$$
\begin{aligned}
\left\|\zeta^{n}\right\|^{2} \leqslant & \frac{c_{2} k}{1-c_{1} k} \sum_{j=0}^{n-1}\left(\frac{1+c_{1} k}{1-c_{1} k}\right)^{j}\left(\left\|\rho^{n-1-j}\right\|^{2}+\left\|\delta^{n-1-j}\right\|^{2}+h^{2 r}\right) \\
& +\left(\frac{1+c_{1} k}{1-c_{1} k}\right)^{n}\left\|\zeta^{0}\right\|^{2},
\end{aligned}
$$

provided $0<1-c_{1} k<1$. 
Now, since

$$
\left(\frac{1+c_{1} k}{1-c_{1} k}\right)^{j} \leqslant\left(\frac{1+c_{1} k}{1-c_{1} k}\right)^{n} \leqslant e^{2 c_{1} T}, \quad 0 \leqslant j \leqslant n-1,
$$

we have, using (3.18) and (2.4), that

$$
\left\|\zeta^{n}\right\|^{2} \leqslant c\left\{k \sum_{j=0}^{n-1}\left(\left\|\rho^{j}\right\|^{2}+\left\|\delta^{j}\right\|^{2}\right)+h^{2 r}\right\} .
$$

From above,

$$
\begin{aligned}
\rho^{n} & =u_{h t}^{n+1 / 2}-\partial u_{h}^{n} \\
& =-\frac{1}{2 k} \int_{n k}^{(n+1) k}[(n+1) k-s][n k-s] u_{h t t t}(x, s) d s .
\end{aligned}
$$

Hence

$$
\begin{aligned}
\left\|\rho^{n}\right\| & \leqslant \frac{1}{2 k} \int_{n k}^{(n+1) k}|[(n+1) k-s][n k-s]|\left\|u_{h t t t}\right\| d s \\
& \leqslant \frac{1}{2 k}\left\{\frac{k^{5}}{30}\right\}^{1 / 2}\left\{\int_{n k}^{(n+1) k}\left\|u_{h t t t}\right\|^{2} d s\right\}^{1 / 2} .
\end{aligned}
$$

Thus

$$
k \sum_{j=0}^{n-1}\left\|\rho^{j}\right\|^{2} \leqslant c k^{4} \sum_{j=0}^{J-1} \int_{j k}^{(j+1) k}\left\|u_{h t t t}\right\|^{2} d s \leqslant c k^{4}\left\|u_{h t t t}\right\|_{L^{2}\left(0, T ; L^{2}(0,1)\right)}
$$

Also

$$
\begin{aligned}
\delta^{n} & =\frac{1}{4}\left[u_{h}^{n+1}-u_{h}^{n}\right]\left[u_{h x}^{n+1}-u_{h x}^{n}\right] \\
& =\frac{1}{4}\left\{\int_{n k}^{(n+1) k} u_{h t}(x, s) d s\right\}\left\{\int_{n k}^{(n+1) k} u_{h t x}(x, s) d s\right\} .
\end{aligned}
$$

Now

$$
\sup _{0 \leqslant x \leqslant 1}\left|\delta^{n}\right| \leqslant \frac{1}{4} k^{2}\left\|u_{h t}\right\|_{L^{\infty}\left(0, T ; L^{\infty}(0,1)\right)}\left\|u_{h t x}\right\|_{L^{\infty}\left(0, T ; L^{\infty}(0,1)\right)}, \quad 0 \leqslant n \leqslant J-1 .
$$

Thus

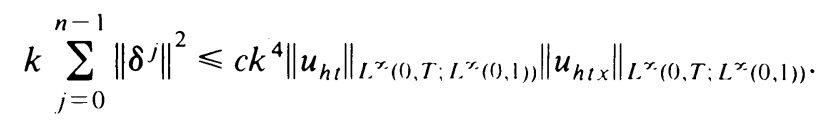

Using (3.31) and (3.32) in (3.30), it follows from (3.21) and (3.22), that, for some constant $c=c(u, T)$,

$$
\left\|\zeta^{n}\right\| \leqslant c\left\{k^{2}+h^{r}\right\}, \quad 0 \leqslant n \leqslant J .
$$

Moreover, $u^{n}-U^{n}=u^{n}-u_{h}^{n}+u_{h}^{n}-U^{n}$ gives

$$
\max _{0 \leqslant n \leqslant J}\left\|u^{n}-U^{n}\right\| \leqslant c\left\{h^{r}+k^{2}\right\}
$$

for some constant $c$ independent of $h$ and $k$. 
We shall next prove the existence of a sequence $\left\{U^{n}\right\}_{n=0}^{J}$ satisfying (2.6). For this, we shall use the following variant of the well-known fixed point theorem of Brouwer (cf. [11, pp. 164-166]).

LEMMA 3.3. Let $H$ be a finite-dimensional Hilbert space with inner product $(\cdot, \cdot)_{H}$, and norm $\|\cdot\|_{H}$. Let the map $g: H \rightarrow H$ be continuous. Suppose there exists $\alpha>0$ such that $(g(Z), Z)_{H}>0$ for all $Z$ with $\|Z\|_{H}=\alpha$. Then there exists $Z^{*} \in H,\left\|Z^{*}\right\|_{H} \leqslant \alpha$ such that $g\left(Z^{*}\right)=0$.

The argument of existence of $\left\{U^{n}\right\}_{n=0}^{J}$ proceeds in an inductive way. Obviously $U^{0}$ exists. Moreover assume $\left\{U^{j}\right\}_{j=0}^{n}$ exists.

For $Z \in S_{h}^{r}$, define $g: S_{h}^{r} \rightarrow S_{h}^{r}$ by

$$
(g(Z), \chi)=\left(Z-2 U^{n}, \chi\right)+\frac{k}{4}\left(Z Z_{x}, \chi\right)+\frac{k}{2}\left(Z_{x x x}, \chi\right), \quad \forall \chi \in S_{h}^{r} .
$$

Such a map exists by the Riesz representation theorem; the fact that $g$ is continuous follows easily from (2.3a) and (2.3b). Furthermore, by periodicity, letting $\chi=Z$

$$
(g(Z), Z)=\left(Z-2 U^{n}, Z\right) \geqslant\|Z\|\left\{\|Z\|-2\left\|U^{n}\right\|\right\} \geqslant\|Z\|\left\{\|Z\|-2\left\|U^{0}\right\|\right\}
$$

from (3.24). Letting $\alpha>2\left\|U^{0}\right\|$, we deduce the existence via Lemma 3.3 of a $Z^{*} \in S_{h}^{r}$ such that $g\left(Z^{*}\right)=0$. Letting $U^{n+1}=Z^{*}-U^{n}$, we get from (3.34) that

$$
\left(U^{n+1}-U^{n}, \chi\right)+k\left(U^{n+1 / 2} U_{x}^{n+1 / 2}+U_{x x x}^{n+1 / 2}, \chi\right)=0, \quad \forall \chi \in S_{h}^{r},
$$

proving the existence of $U^{n+1}$.

For uniqueness, suppose that $V^{n+1} \in S_{h}^{r}$ satisfies

$$
\left(\partial V^{n}+V^{n+1 / 2} V_{x}^{n+1 / 2}+V_{x x x}^{n+1 / 2}, \chi\right)=0, \quad \forall \chi \in S_{h}^{r} .
$$

Letting $E^{i}=U^{i}-V^{i}, i=n, n+1$, from (2.6) and (3.35) we have for $\chi \in S_{h}^{r}$,

$$
\begin{aligned}
& \left(\partial E^{n}+E_{x x x}^{n+1 / 2}, \chi\right) \\
& \quad=\left(E^{n+1 / 2} E_{x}^{n+1 / 2}-U_{x}^{n+1 / 2} E^{n+1 / 2}-U^{n+1 / 2} E_{x}^{n+1 / 2}, \chi\right) .
\end{aligned}
$$

Letting $x=E^{n+1 / 2}$ in (3.36),

$$
\begin{aligned}
\left\|E^{n+1}\right\|^{2}- & \left\|E^{n}\right\|^{2}=-k\left(U_{x}^{n+1 / 2},\left[E^{n+1 / 2}\right]^{2}\right) \\
& \leqslant k\left\|U_{x}^{n+1 / 2}\right\|_{L^{\infty}(0,1)}\left\|E^{n+1 / 2}\right\|^{2} \\
& \leqslant \frac{k}{2}\left\{\left\|u_{h x}^{n+1 / 2}\right\|_{L^{\infty}(0,1)}+\left\|u_{h x}^{n+1 / 2}-U_{x}^{n+1 / 2}\right\|_{L^{\infty}(0,1)}\right\}\left\{\left\|E^{n+1}\right\|^{2}+\left\|E^{n}\right\|^{2}\right\} \\
& \leqslant c\left\{k+k h^{r-3 / 2}+k^{3} h^{-3 / 2}\right\}\left\{\left\|E^{n+1}\right\|^{2}+\left\|E^{n}\right\|^{2}\right\}
\end{aligned}
$$

from (3.22), (3.33), and (2.3b).

Hence, for $k, k h^{-1 / 2}$ sufficiently small, we get

$$
\left\|E^{n+1}\right\| \leqslant \lambda\left\|E^{n}\right\|
$$

where

$$
\lambda=\left\{\frac{1+c k+c k^{3} h^{-3 / 2}}{1-c k-c k^{3} h^{-3 / 2}}\right\}^{1 / 2}
$$


Now taking $V^{n}=U^{n}$, we see that $E^{n+1}=0$, hence uniqueness follows. Obviously the condition " $k h^{-1 / 2}$ sufficiently small" may be replaced in the above proof by the requirements that $k$ be sufficiently small and $k h^{-3 / 4}$ remain bounded as $k, h \rightarrow 0$.

Before proceeding with the proof of Theorem 2.3, we motivate the construction of (2.10). For this, let $G: S_{h}^{r} \rightarrow S_{h}^{r}$ be defined by

$$
\begin{aligned}
(G(\phi), \chi)= & \left(\phi-\tilde{U}_{j_{n}}^{n}, \chi\right)-\frac{k}{8}\left(\phi^{2}+2 \phi \tilde{U}_{j_{n}}^{n}+\left[\tilde{U}_{j_{n}}^{n}\right]^{2}, \chi_{x}\right) \\
& +\frac{k}{2}\left(\phi_{x x x}+\left[\tilde{U}_{j_{n}}^{n}\right]_{x x x}, \chi\right), \quad \phi \in S_{h}^{r}, \chi \in S_{h}^{r} .
\end{aligned}
$$

Such a map exists by the Riesz representation theorem and is continuous. For $z \in S_{h}^{r}$, the Gâteaux derivative of $G$ in the direction $\phi$ evaluated at $z$ is given by

$$
\left(\mathcal{G}_{[z]}(\phi), \chi\right)=(\phi, \chi)-\frac{k}{4}\left(\phi z, \chi_{x}\right)-\frac{k}{4}\left(\phi \tilde{U}_{j_{n}}^{n}, \chi_{x}\right)+\frac{k}{2}\left(\phi_{x x x}, \chi\right), \quad \forall \chi \in S_{h}^{r} \text {. }
$$

Thus, given $\tilde{U}_{0}^{n+1}$, we define the sequence of Newton iterants $\left\{\tilde{U}_{j}^{n+1}\right\}_{j=0}^{j_{n+1}}$ by

$$
\left(\mathcal{G}_{\left[\tilde{U}_{j}^{n+1}\right]}\left(\tilde{U}_{j+1}^{n+1}-\tilde{U}_{j}^{n+1}\right), \chi\right)=-\left(G\left(\tilde{U}_{j}^{n+1}\right), \chi\right), \quad \forall \chi \in S_{h}^{r}, 0 \leqslant j \leqslant j_{n+1}-1,
$$

which is equivalent to (2.10).

Proof of Theorem 2.3. We begin by making the following assumption: Let $C_{-1}^{*}$, $C_{-2}^{*}$ be nonnegative constants independent of $h$ and $k$,

Induction Hypothesis I (on $n$ ).

(a) $\tilde{U}_{j_{1}}^{i}$ exists uniquely, $0 \leqslant i \leqslant n$,

(b) $\left\|U^{i}-\tilde{U}_{j_{i}}^{i}\right\| \leqslant C_{i}^{*}\left\{k^{2}+h^{r}\right\}, 0 \leqslant i \leqslant n$,

(c) $C_{i}^{*}=C k+(1+C k) C_{i-1}^{*}+C k C_{i-2}^{*}, 0 \leqslant i \leqslant n$,

where the constant $C$, to be suitably chosen later, is independent of $n, h, k$ and the integers $j_{i}, 0 \leqslant i \leqslant n$. Note that, since we have taken $\tilde{U}_{j_{0}}^{0}=U^{0}=P u^{0}$, (a)-(c) hold for $n=0$. Moreover, if (c) holds for $0 \leqslant i \leqslant n$, then it is easily shown that the constants $\left\{C_{i}^{*}\right\}_{i=0}^{n}$ are uniformly bounded by a constant $C^{*}$ independent of $n, h, k$ and the integers $j_{i}$.

We next show that $\tilde{U}^{n+1}$ defined by (2.7) exists uniquely. Its existence follows from Lemma 3.3 (just replace $U^{n}$ by $\tilde{U}_{j_{n}}^{n}$ in (3.34)). To show that $\tilde{U}^{n+1}$ is unique, note first that by letting $V^{n}=\tilde{U}_{j_{n}}^{n}, V^{n+1}=\tilde{U}^{n+1}$ in (3.35), we get from (3.37)

$$
\left\|U^{n+1}-\tilde{U}^{n+1}\right\| \leqslant \lambda\left\|U^{n}-\tilde{U}_{j_{n}}^{n}\right\|
$$

provided $k h^{-1 / 2}$ is sufficiently small. Now suppose there exists $\tilde{V}^{n+1} \in S_{h}^{r}$ satisfying

$$
\begin{aligned}
\left(\tilde{V}^{n+1}-\tilde{U}_{j_{n}}, \chi\right) & -\frac{k}{8}\left(\left[\tilde{V}^{n+1}+\tilde{U}_{j_{n}}^{n}\right]^{2}, \chi_{x}\right) \\
+ & \frac{k}{2}\left(\left[\tilde{V}^{n+1}+\tilde{U}_{j_{n}}^{n}\right]_{x x x}, \chi\right)=0, \quad \forall \chi \in S_{h}^{r} .
\end{aligned}
$$

Letting $\tilde{\zeta}^{n+1}=\tilde{U}^{n+1}-\tilde{V}^{n+1}$, from (2.7) and (3.40) we get

$$
\begin{aligned}
\left(\tilde{\zeta}^{n+1}+\frac{k}{2} \tilde{\zeta}_{x x x}^{n+1}, \chi\right) & =\frac{k}{8}\left(\left[\tilde{U}^{n+1}+\tilde{U}_{j_{n}}^{n}\right]^{2}, \chi_{x}\right)-\frac{k}{8}\left(\left[\tilde{V}^{n+1}+\tilde{U}_{j_{n}}^{n}\right]^{2}, \chi_{x}\right) \\
& =\frac{k}{4}\left(\left[\tilde{U}^{n+1}+\tilde{U}_{j_{n}}^{n}\right] \tilde{\zeta}^{n+1}, \chi_{x}\right)-\frac{k}{8}\left(\left[\tilde{\zeta}^{n+1}\right]^{2}, \chi_{x}\right) .
\end{aligned}
$$


Letting $\chi=\tilde{\zeta}^{n+1}$ in (3.41), we get

$$
\left\|\tilde{\zeta}^{n+1}\right\|^{2}=-\frac{k}{8}\left(\left[\tilde{U}^{n+1}+\tilde{U}_{j_{n}}^{n}\right]_{x},\left[\tilde{\zeta}^{n+1}\right]^{2}\right)
$$

Hence

$$
\left\|\tilde{\zeta}^{n+1}\right\|^{2}\left\{1-\frac{k}{8}\left\|\left[\tilde{U}^{n+1}+\tilde{U}_{j_{n}}^{n}\right]_{x}\right\|_{L^{x}(0,1)}\right\} \leqslant 0
$$

Now

$$
\begin{aligned}
\left\|\left[\tilde{U}^{n+1}+\tilde{U}_{j_{n}}^{n}\right]_{x}\right\|_{L^{\infty}(0,1)} \leqslant & \left\|u_{h x}^{n+1}+u_{h x}^{n}\right\|_{L^{\infty}(0,1)}+\left\|\left[\tilde{U}^{n+1}-U^{n+1}\right]_{x}\right\|_{L^{\infty}(0,1)} \\
& +\left\|\left[U^{n+1}-u_{h}^{n+1}\right]_{x}\right\|_{L^{\infty}(0,1)}+\left\|\left[\tilde{U}_{j_{n}}^{n}-U^{n}\right]_{x}\right\|_{L^{\infty}(0,1)} \\
& +\left\|\left[U^{n}-u_{h}^{n}\right]_{x}\right\|_{L^{\infty}(0,1)} .
\end{aligned}
$$

It follows from (3.22), (3.33), (3.39), (2.3b), and part (b) of Hypothesis I that

$$
k\left\|\left[\tilde{U}^{n+1}+\tilde{U}_{j_{n}}^{n}\right]_{x}\right\|_{L^{\infty}(0,1)} \leqslant c k+c k^{3} h^{-3 / 2}+c k\left(k^{2}+h^{r}\right) h^{-3 / 2}(\lambda+1) C_{n}^{*} .
$$

Hence, e.g. for $k$ and $k h^{-1 / 2}$ sufficiently small, the coefficient of $\left\|\tilde{\zeta}^{n+1}\right\|^{2}$ in (3.42) is positive, thus $\zeta^{n+1}=0$, proving uniqueness of $\tilde{U}^{n+1}$.

Our next task is to show that the initial iterants $\tilde{U}_{0}^{n+1}$ given by (2.9) and (2.9') are well defined and are good approximations to $\tilde{U}^{n+1}$. We first deal with the case $n=0$. Note that by (2.7) and (2.8), $\tilde{U}^{1}=U^{1}$. Now $\tilde{U}_{0}^{1}$ is given as the solution of a linear system of equations, with associated bilinear form

$$
\mathbb{Q}(\phi, \chi)=\left(\phi+k U^{0} \phi_{x}+k \phi_{x x x}, \chi\right), \quad \phi, \chi \in S_{h}^{r} .
$$

Hence with $\phi=\chi$

$$
\mathbb{Q}(\phi, \phi)=\|\phi\|^{2}-\frac{k}{2}\left(U_{x}^{0}, \phi^{2}\right) \geqslant\|\phi\|^{2}\left[1-\frac{k}{2}\left\|U_{x}^{0}\right\|_{L^{\infty}(0,1)}\right],
$$

and by (2.4), (2.3b) $\tilde{U}_{0}^{1}$ exists uniquely (for $k$ sufficiently small). Also, it is easy to show (with methods of estimation similar to those used in the proof of Theorem 2.2) that there exists a constant $\bar{c}_{1}$, independent of $h$ and $k$, such that

$$
\left\|\tilde{U}_{0}^{1}-\tilde{U}^{1}\right\|=\left\|\tilde{U}_{0}^{1}-U^{1}\right\| \leqslant \bar{c}_{1}\left\{k^{2}+h^{r}\right\} .
$$

Also, for $n \geqslant 1$, we have

$$
\begin{aligned}
\tilde{U}^{n+1}-\tilde{U}_{0}^{n+1}=\tilde{U}^{n+1}-\left[2 \tilde{U}_{j_{n}}^{n}-\tilde{U}_{j_{n-1}}^{n-1}\right] \\
=\left[\tilde{U}^{n+1}-U^{n+1}\right]+\left[U^{n+1}-u_{h}^{n+1}\right]+\left[u_{h}^{n+1}-2 u_{h}^{n}+u_{h}^{n-1}\right] \\
\quad+2\left[u_{h}^{n}-U^{n}\right]-\left[u_{h}^{n-1}-U^{n-1}\right]+2\left[U^{n}-\tilde{U}_{j_{n}}^{n}\right]-\left[U^{n-1}-\tilde{U}_{j_{n-1}}^{n-1}\right] .
\end{aligned}
$$

Now since $\left\|u_{h}^{n+1}-2 u_{h}^{n}+u_{h}^{n-1}\right\| \leqslant c k^{2}$ by (3.21), it follows from (3.33), (3.39) and part (b) of Hypothesis I that

$$
\left\|\tilde{U}^{n+1}-\tilde{U}_{0}^{n+1}\right\| \leqslant\left\{c+(\lambda+2) C_{n}^{*}+C_{n-1}^{*}\right\}\left(k^{2}+h^{r}\right) .
$$

We let

$$
\bar{c}_{n+1}=c+(\lambda+2) C_{n}^{*}+C_{n-1}^{*}, \quad n \geqslant 1 .
$$


Note that, for $k h^{-1 / 2}$ sufficiently small, $\bar{c}_{n+1}$ is bounded above by a constant independent of $h, k, j_{i}$, and $n$. Also note that the constant $c$ in (3.46) does not depend on $C$ of Hypothesis I, part (c).

We next show that there exists a unique sequence $\left\{\tilde{U}_{j}^{n+1}\right\}_{j=1}^{j_{n+1}}$ satisfying (2.10). For this, we give the following "internal" inductive proof.

Induction Hypothesis II (on $j$ ).

(a) $\tilde{U}_{m}^{n+1}$ exists uniquely for $0 \leqslant m \leqslant j \leqslant j_{n+1}-1$,

(b) $\left\|\tilde{U}^{n+1}-\tilde{U}_{m}^{n+1}\right\| \leqslant \bar{c}_{n+1}\left(k+h^{r / 2}\right)^{2^{m}+1}$ for $0 \leqslant m \leqslant j \leqslant j_{n+1}-1$.

It follows from (3.44) and (3.45) that Hypothesis II holds for $j=0$. We now show that it also holds for $j+1 . \tilde{U}_{j+1}^{n+1}$ is given as the solution of a linear system of equations. The associated bilinear form is

$$
\Re(\phi, \chi)=\left(\phi+\frac{k}{2} \phi_{x x x}, \chi\right)-\frac{k}{4}\left(\left[\tilde{U}_{j}^{n+1}+\tilde{U}_{j_{n}}^{n}\right] \phi, \chi_{x}\right), \quad \phi, \chi \in S_{h}^{r} .
$$

Now

$$
\begin{aligned}
\mathscr{B}(\phi, \phi) & =\|\phi\|^{2}+\frac{k}{8}\left(\left[\tilde{U}_{j}^{n+1}+\tilde{U}_{j_{n}}^{n}\right]_{x}, \phi^{2}\right) \\
& \geqslant\|\phi\|^{2}\left\{1-\frac{k}{8}\left\|\left[\tilde{U}_{j}^{n+1}+\tilde{U}_{j_{n}}^{n}\right]_{x}\right\|_{L^{\infty}(0,1)}\right\} .
\end{aligned}
$$

From (3.43) and part (b) of Hypothesis II, we have

$$
\begin{aligned}
k\left\|\left[\tilde{U}_{j}^{n+1}+\tilde{U}_{j_{n}}^{n}\right]_{x}\right\|_{L^{\infty}(0,1)} & =k\left\|\left[\tilde{U}_{j}^{n+1}-\tilde{U}^{n+1}\right]_{x}\right\|_{L^{\infty}(0,1)}+k\left\|\left[\tilde{U}^{n+1}+\tilde{U}_{j_{n}}^{n}\right]_{x}\right\|_{L^{\infty}(0,1)} \\
\leqslant & c k h^{-3 / 2} \bar{c}_{n+1}\left(k+h^{r / 2}\right)^{2^{j}+1}+c k+c k^{3} h^{-3 / 2} \\
& +c\left(k^{3}+k h^{r}\right) h^{-3 / 2}(\lambda+1) C_{n}^{*} .
\end{aligned}
$$

Now since $j \geqslant 0$, it follows from (3.48) and (3.46) that, for $k, k h^{-1 / 2}$ sufficiently small, the coefficient of $\|\phi\|^{2}$ in (3.47) is positive; hence $\tilde{U}_{j+1}^{n+1}$ exists uniquely.

Subtracting (2.7) from the left-hand side of (2.10), we get

$$
\begin{aligned}
\left(\left[\tilde{U}_{j+1}^{n+1}-\tilde{U}^{n+1}\right]\right. & \left.+\frac{k}{2}\left[\tilde{U}_{j+1}^{n+1}-\tilde{U}^{n+1}\right]_{x x x}, \chi\right) \\
& -\frac{k}{4}\left(\left[\tilde{U}_{j}^{n+1}+\tilde{U}_{j_{n}}^{n}\right]\left[\tilde{U}_{j+1}^{n+1}-\tilde{U}^{n+1}\right], \chi_{x}\right) \\
= & -\frac{k}{8}\left(\left[\tilde{U}_{j}^{n+1}-\tilde{U}^{n+1}\right]^{2}, \chi_{x}\right), \quad \forall \chi \in S_{h}^{r} .
\end{aligned}
$$

Letting $\chi=\tilde{U}_{j+1}^{n+1}-\tilde{U}^{n+1}$ in (3.49), we get

$$
\begin{aligned}
\left\|\tilde{U}_{j+1}^{n+1}-\tilde{U}^{n+1}\right\|^{2}+\frac{k}{8}\left(\left[\tilde{U}_{j}^{n+1}\right.\right. & \left.\left.+\tilde{U}_{j_{n}}^{n}\right]_{x},\left[\tilde{U}_{j+1}^{n+1}-\tilde{U}^{n+1}\right]^{2}\right) \\
& =-\frac{k}{8}\left(\left[\tilde{U}_{j}^{n+1}-\tilde{U}^{n+1}\right]^{2},\left[\tilde{U}_{j+1}^{n+1}-\tilde{U}^{n+1}\right]_{x}\right) .
\end{aligned}
$$

Hence

$$
\begin{aligned}
\left\|\tilde{U}_{j+1}^{n+1}-\tilde{U}^{n+1}\right\|\left\{1-\frac{k}{8}\left\|\left[\tilde{U}_{j}^{n+1}+\tilde{U}_{j_{n}}^{n}\right]_{x}\right\|_{L^{\infty}(0,1)}\right\} \\
\quad \leqslant c k h^{-3 / 2}\left\|\tilde{U}_{j}^{n+1}-\tilde{U}^{n+1}\right\|^{2} .
\end{aligned}
$$


It follows from (3.48), (3.50) and part (b) of Hypothesis II that for $k, k h^{-1 / 2}$ sufficiently small

$$
\begin{aligned}
\left\|\tilde{U}_{j+1}^{n+1}-\tilde{U}^{n+1}\right\| & \leqslant c k h^{-3 / 2}\left\|\tilde{U}_{j}^{n+1}-\tilde{U}^{n+1}\right\|^{2} \\
& \leqslant\left(c k h^{-3 / 2}\right)^{2^{j+1}-1}\left\|\tilde{U}_{0}^{n+1}-\tilde{U}^{n+1}\right\|^{2^{j+1}} \\
& \leqslant\left[c \bar{c}_{n+1} k h^{-3 / 2}\left(k+h^{r / 2}\right)\right]^{2^{j+1}-1} \bar{c}_{n+1}\left(k+h^{r / 2}\right)^{2^{j+1}+1} .
\end{aligned}
$$

Now, for $k, k h^{-3 / 4}$ sufficiently small, $c \bar{c}_{n+1}\left(k^{2} h^{-3 / 2}+k h^{(r-3) / 2}\right) \leqslant 1$. Hence

$$
\left\|\tilde{U}_{j+1}^{n+1}-\tilde{U}^{n+1}\right\| \leqslant \bar{c}_{n+1}\left(k+h^{r / 2}\right)^{2^{j+1}+1},
$$

completing the induction argument II.

It remains to complete the induction argument I. We distinguish two cases. First assume that $h^{r / 2} \leqslant k$. Then from (3.39), (3.45), (3.52) we obtain, since $j_{n+1} \geqslant 1$,

$$
\begin{aligned}
\left\|U^{n+1}-\tilde{U}_{j_{n+1}}^{n+1}\right\| & \leqslant\left\|U^{n+1}-\tilde{U}^{n+1}\right\|+\left\|\tilde{U}^{n+1}-\tilde{U}_{j_{n+1}+1}^{n+1}\right\| \\
& \leqslant \lambda C_{n}^{*}\left(k^{2}+h^{r}\right)+\bar{c}_{n+1}\left(k+h^{r / 2}\right)^{2^{j_{n+1}+1}} \\
& \leqslant\left\{(\lambda+4 k \lambda+8 k) C_{n}^{*}+4 c k+4 k C_{n-1}^{*}\right\}\left(k^{2}+h^{r}\right),
\end{aligned}
$$

where the $c$ occurring in the right-hand side of the above is independent of $C$ of part (c) of the Induction Hypothesis I. Now, it can be arranged a priori, in view of (3.38), that $k$ and $k h^{-3 / 4}$ be taken sufficiently small so that for some $c$ independent of $C$ we have

$$
\lambda \leqslant 1+c k
$$

Hence (3.53) becomes

$$
\left\|U^{n+1}-\tilde{U}_{j_{n+1}}^{n+1}\right\| \leqslant\left[c k+(1+c k) C_{n}^{*}+4 k C_{n-1}^{*}\right]\left(k^{2}+h^{r}\right),
$$

which, with the choice $C=\max (c, 4)$ defines $C_{n+1}^{*}$ satisfying part (c) of the Induction Hypothesis I and completes the inductive step.

If now it is the case that $k<h^{r / 2}$, we obtain, by (3.51) for $0 \leqslant j \leqslant j_{n+1}$, that

$$
\left\|\tilde{U}_{j}^{n+1}-\tilde{U}^{n+1}\right\| \leqslant \bar{c}_{n+1} k^{2^{j}-1}\left(k+h^{r / 2}\right)^{2^{j+1}}\left[2 c \bar{c}_{n+1} h^{(r-3) / 2}\right]^{2^{j}-1} .
$$

Since $r \geqslant 4,2 c \bar{c}_{n+1} h^{(r-3) / 2}$ can be made less than or equal to 1 by choosing eventually $h$ sufficiently small. So we either revert to the previous case $h^{r / 2} \leqslant k$ or we obtain, for $0 \leqslant j \leqslant j_{n+1}$, that

$$
\left\|\tilde{U}_{j}^{n+1}-\tilde{U}^{n+1}\right\| \leqslant \bar{c}_{n+1} k^{2^{j-1}}\left(k+h^{r / 2}\right)^{2^{j+1}} .
$$

Hence, by similar estimates to those used in the derivation of (3.53) we see, since $j_{n+1} \geqslant 1$, that

$$
\begin{aligned}
\left\|U^{n+1}-\tilde{U}_{j_{n+1}+1}^{n+1}\right\| & \leqslant \lambda C_{n}^{*}\left(k^{2}+h^{r}\right)+k^{2^{j_{n+1}}-1} \bar{c}_{n+1}\left(k+h^{r / 2}\right)^{2^{j_{n+1}+1}} \\
& \leqslant\left(\lambda C_{n}^{*}+2 k \bar{c}_{n+1}\right)\left(k^{2}+h^{r}\right) \\
& \leqslant\left[(\lambda+2 k \lambda+4 k) C_{n}^{*}+2 c k+2 k C_{n-1}^{*}\right]\left(k^{2}+h^{r}\right) .
\end{aligned}
$$

Since (3.54) may have been arranged a priori to hold by taking $k$ and $k h^{-3 / 4}$ sufficiently small, we are led again to a choice of $C$ and the completion of the 
inductive step. (Note that a hypothesis of the form $k \geqslant h^{r / 2}$, for all $k, h$ sufficiently small, is not restrictive and certainly gives a nonempty interval of time steps $k$ that also satisfy $k \leqslant \alpha h^{3 / 4}$ for any $\alpha>0$. Hence, if the "boundedness below" assumption $k h^{-r / 2} \geqslant 1$ is also imposed in the statement of the theorem, the inductive step is completed just by (3.53)-(3.55).)

Laboratorio de Computacao Cientificas-CNPq

Av. Wenceslau Braz 71

22290 Rio de Janeiro-R. J., Brazil

Department of Mathematics

The University of Tennessee

Knoxville, Tennessee 37996

Department of Mathematics

The University of Tennessee

Knoxville, Tennessee 37996

1. K. ABE \& O. INOUE, "Fourier expansion solution of the Korteweg-de Vries equation," J. Comput. Phys., v. 34, 1980, pp. 202-210.

2. M. E. Alexander \& J. LL. Morris, "Galerkin methods applied to some model equations for nonlinear dispersive waves," J. Comput. Phys., v. 30, 1979, pp. 428-451.

3. J. BONA \& R. SCort, "Solutions of the Korteweg-de Vries equation in fractional order Sobolev spaces," Duke Math. J., v. 43, 1976, pp. 87-99.

4. J. Bona \& R. Smith, "The initial value problem for the Korteweg-de Vries equation," Philos. Trans. Roy. Soc. London Ser. A, v. 278, 1975, pp. 555-604.

5. B. FornBerg \& G. B. Whitham, "A numerical and theoretical study of certain nonlinear wave phenomena," Philos. Trans. Roy. Soc. London Ser. A, v. 289, 1978, pp. 373-404.

6. I. S. Greig \& J. LL. Morris, "A Hopscotch method for the Korteweg-de Vries equation," $J$. Comput. Phys., v. 20, 1976, pp. 64-80.

7. P. D. LAX, "Almost periodic solutions of the Korteweg-de Vries equation," SIAM Rev., v. 18, 1976, pp. 351-375.

8. H. SCHAmel \& K. ElsäSSER, "The application of the spectral method to nonlinear wave propagation," J. Comput. Phys., v. 22, 1976, pp. 501-516.

9. F. TAPPERT, "Numerical solutions of the Korteweg-de Vries equation and its generalizations by the split-step Fourier method," in Nonlinear Wave Motion (A. C. Newell, Ed.), Lectures in Appl. Math., Vol. 15. Amer. Math. Soc., Providence, R.I., 1974, pp. 215-216.

10. R. Temam, "Sur un problème non linéaire," J. Math. Pures Appl., v. 48, 1969, pp. 159-172.

11. R. Temam, Navier-Stokes Equations: Theory and Numerical Analysis, rev. ed., North-Holland, Amsterdam, 1979.

12. V. THOMEE \& B. WeNDROFF, "Convergence estimates for Galerkin methods for variable coefficient initial value problems," SIAM J. Numer. Anal., v. 11, 1974, pp. 1059-1068.

13. A. C. Vliegenhart, "On finite-difference methods for the Korteweg-de Vries equation," J. Engrg. Math., v. 5, 1971, pp. 137-155.

14. L. B. WAHLBIN, "A dissipative Galerkin method for the numerical solution of first order hyperbolic equations," in Mathematical Aspects of Finite Elements in Partial Differential Equations (C. de Boor, Ed.), Academic Press, New York, 1974, pp. 147-169.

15. R. WINTHER, "A conservative finite element method for the Korteweg-de Vries equation," Math. Comp., v. 34, 1980, pp. 23-43.

16. N. J. Zabusky, "Computation: Its role in mathematical physics innovation," J. Comput. Phys., v. 43, 1981, pp. 195-249.

17. N. J. ZabusKY \& M. D. KruSKal, "Interaction of "solitons" in a collisionless plasma and the recurrence of initial states," Phys. Rev. Lett., v. 15, 1965, pp. 240-243. 